\title{
Treinamento auditivo computadorizado em uma adolescente portadora de neurofibromatose do tipo 2
}

\author{
Computerized auditory training in an \\ adolescent with neurofibromatosis type 2
}

\section{Entrenamiento auditivo computarizado en una adolescente portadora de neurofibromatosis tipo 2}

\author{
Eduarda Pazini* \\ Pâmela Mariel Marques* \\ Ayra Renata D'Agostini* \\ Bianca Bertuol* \\ Themis Maria Kessler* \\ Michele Vargas Garcia* \\ Eliara Pinto Vieira Biaggio*
}

\section{Resumo}

A neurofibromatose do tipo 2 é uma doença genética que afeta o Sistema Nervoso Central, principalmente a via auditiva, sendo alguns dos principais sintomas a perda auditiva neurossensorial, o baixo reconhecimento de fala, zumbido e disfunção vestibular. Relata-se neste estudo um processo de intervenção terapêutica por meio do Treinamento Auditivo Computadorizado em uma adolescente com diagnóstico médico de Neurofibromatose tipo 2. Tal intervenção contou com 16 sessões de TA,

* Universidade Federal de Santa Maria, Santa Maria, Rio Grande do Sul, Brasil

Contribuição dos autores:

EP e ARD: Coleta e análise de dados, redação

PMM: Análise de dados, redação

BB: Redação

TMK e EPVB: Orientação

EPVB: Redação e Orientação

E-mail para correspondência: Eduarda Pazini duda_pazini@hotmail.com

Recebido: 04/12/2017

Aprovado: 03/07/2018 
realizadas com um software específico. Para o estudo realizou-se a avaliação, pré e pós-treinamento, por meio dos resultados da avaliação audiológica básica, Potencial Evocado Auditivo de Tronco Encefálico e avaliação da consciência fonológica, além de analisar os relatos referentes ao processo terapêutico. Os testes comportamentais de processamento auditivo não foram realizados, pois a paciente apresenta acentuada dificuldade de compreensão de fala. Paciente apresentou melhora no reconhecimento de fala e na habilidade de consciência fonológica, apesar do exame eletrofisiológico da audição não demonstrar tais benefícios diretamente na via auditiva em nível de tronco encefálico, para o estímulo clique. Destaca-se o relato da paciente de melhora na compreensão de fala após tal intervenção. Desse modo, observaram-se os efeitos positivos do treinamento auditivo na melhora da percepção auditiva, influenciando positivamente na vida diária da paciente.

Palavras-chave: Schwannoma; Percepção Auditiva; Audiologia; Fonoaudiologia; Qualidade de vida; Neurofibromatose 2.

\section{Abstract}

Neurofibromatosis type 2 is a genetic disease that affects the Central Nervous System, mainly the auditory pathway, being some of the main symptoms: sensorineural hearing loss, low speech recognition, tinnitus and vestibular dysfunction. This study describes a process of therapeutic intervention through Computerized Auditory Training in an adolescent with medical diagnosis of Neurofibromatosis type 2. This intervention was composed of 16 sessions of AT with a specific software. For this study, the pre and post-training evaluation was carried out through the results of basic audiological evaluation, Brainstem Evoked Response Audiometry and evaluation of phonological awareness, as well as analyzing the patient's reports regarding the therapeutic process. The auditory processing behavioral tests were not performed, since the patient presents difficulty in speech comprehension. Patient presented improvement in speech recognition and phonological awareness ability, although the electrophysiological test did not demonstrate such benefits directly in the auditory pathway at the brainstem level, for the click stimulus. Furthermore, the patient's report of improvement in speech comprehension after such intervention could also be highlighted. Thus, the positive effects of auditory training on the improvement of auditory perception could be observed, influencing positively the daily life of the patient.

Keywords: Schwannoma; Auditory Perception; Audiology; Speech Language Pathology and Audiology Therapy; Quality of life; Neurofibromatosis 2.

\section{Resumen}

La neurofibromatosis del tipo 2 es una enfermedad genética que afecta el Sistema Nervioso Central, principalmente la vía auditiva,siendo algunos de los principales síntomas la pérdida auditiva neurosensorial, el bajo reconocimiento de habla, zumbido e disfunción vestibular. En este estudio se relató un proceso de intervención terapéutica por medio de Entrenamiento Auditivo Computarizado en una adolescente diagnosticada con Neurofibromatosis tipo 2. La intervención contó con 16 sesiones de EA, realizadas con un software específico. Para el estudio se realizó la evaluación antes y después del entrenamiento, atreves de los resultados de la evaluación audiológica básica, Potenciales Evocados Auditivos del Tronco Encefálico y evaluación de la conciencia fonológica, además de analizar los relatos relacionados al proceso terapéutico. Los testes comportamentales de procesamiento auditivo no fueron realizados, pues la paciente presenta acentuada dificultad de comprensión de habla. La paciente presentó mejora en el reconocimiento de habla y en la habilidad de conciencia fonológica, por más que el examen electrofisiológico de la audición no tenga demostrado esos beneficios directamente en la vía auditiva en nivel de tronco encefálico, para el estímulo clic. Se destaca el relato de mejorapor la paciente en la comprensión de habla tras la intervención. De esa manera, se observaron los efectos positivos del entrenamiento auditivo en la mejora de la percepción auditiva, influyendo positivamente en la vida diaria de la paciente.

Palabras claves: Schwannoma; Percepción Auditiva; Audiología; Fonoaudiología; Calidad de vida; Neurofibromatosis 2. 


\section{Introdução}

A Neurofibromatose (NF) é uma doença genética, cuja manifestação e severidade variam em cada paciente, com múltiplas manifestações clínicas, sendo os principais sintomas: perda auditiva neurossensorial; baixo reconhecimento e compreensão de fala, especialmente em ambientes ruidosos; zumbido e disfunção vestibular ${ }^{1,2}$. Difere-se da Doença de Meniére por apresentar sintomas contínuos e não apenas em crises. Pode ser classificada em NF do tipo 1 ou tipo 2, sendo que esta última acomete o sistema nervoso central e é caracterizada pela presença do Schwannoma do VIII par craniano, geralmente bilateral ${ }^{3}$.

O Schwannoma do VIII é assim denominado por proliferar-se nas células de Schwann, sendo também conhecido como neurinoma acústico. Acomete o ângulo pontocerebelar, o qual se localiza na fossa posterior cerebelar, onde se encontram porções dos nervos cranianos V, VII, VIII, IX, X e $\mathrm{XI}{ }^{1}$. Caracteriza-se por ser um tumor benigno, de crescimento lento, que acomete o conduto auditivo interno, evoluindo para a cisterna pontina. Em estágios mais avançados, pode comprimir o cerebelo e até mesmo o tronco cerebral ${ }^{3}$.

O diagnóstico desta patologia é feito por meio de testes eletrofisiológicos e comportamentais da audição - como audiometria tonal liminar, índice percentual de reconhecimento de fala, reflexos acústicos estapedianos e Potencial Evocado Auditivo de Tronco Encefálico - além de exames vestibulares como a Eletronistagmografia, exames de imagem, como a ressonância magnética do crânio e a tomografia computadorizada do osso temporal, indicados pelo médico responsável ${ }^{3}$.

Em relação à audição, frequentemente encontram-se valores discrepantes entre o grau da perda auditiva e o reconhecimento de fala, bem como diminuição nas habilidades de percepção auditiva para os sons da fala e de suas particularidades acústicas, ocasionando em limitações quanto aos meios sociais, culturais e emocionais. É possível encontrar também casos de perda auditiva súbita ou plenitude auricular associada à perda flutuante, além das alterações já referenciadas nos exames eletrofisiológicos da audição ${ }^{3}$.

O tratamento deve considerar o tempo de diagnóstico do tumor e as particularidades de cada caso - como tamanho e localização, por exemplo -, sendo que a cirurgia é ainda um recurso bastante empregado para os casos de Schwannoma no VIII par $^{3}$.

Ambos os tipos de NF podem estar associados a alterações das habilidades auditivas, em especial a compreensão de fala, podendo ser observado um transtorno do processamento auditivo (TPA) que pode ser resultante de um evento pós-natal (trauma neurológico ou infecções) ${ }^{4,5}$.

Para tal transtorno, o treinamento auditivo (TA) pode ser um recurso terapêutico empregado visando promover mudanças nas bases neurais e no comportamento auditivo, favorecendo a plasticidade cerebral e, assim, melhora nas habilidades auditivas dos sujeitos ${ }^{6}$.

Considerando o baixo reconhecimento de fala dos sujeitos diagnosticados com Schwannoma, e as dificuldades em processar as informações auditivas por conta do comprometimento no VIII par, hipotetizou-se que um treinamento auditivo poderia ser indicado em um sujeito com Neurofibromatose tipo 2, auxiliando no tratamento e em especial na compreensão de fala, tanto no pré, quanto pós-operatório.

Sendo assim, o objetivo do presente trabalho foi descrever os efeitos do treinamento auditivo computadorizado em uma adolescente diagnosticada com Neurofibromatose tipo 2.

\section{Apresentação de caso}

Estudo de caso desenvolvido no Ambulatório de Audiologia e de Processamento Auditivo de uma Instituição de Ensino Superior, no período de março a junho de 2016. O sujeito desta pesquisa assinou o Termo de Consentimento Livre e Esclarecido (TCLE), respeitando as questões éticas propostas pela Resolução 466/2012 do Conselho Nacional de Saúde. O estudo foi aprovado pelo Comitê de Ética em Pesquisa da referida Instituição, sob o número 43171715.0.0000.5346.

Participou da pesquisa uma adolescente do gênero feminino, 18 anos, diagnosticada com Neurofibromatose do tipo 2. O médico responsável solicitou os exames de Audiometria Tonal Liminar, Logoaudiometria, Imitanciometria e Potencial Evocado Auditivo de Tronco Encefálico (PEATE), pois a paciente apresentava queixas de perda auditiva e considerável dificuldade de compreensão da fala.

Realizou-se Avaliação Audiológica Básica, que envolveu a inspeção visual do Meato Acústico Externo, a fim de verificar a presença de algum 
comprometimento de orelha externa, com o otoscópio Standard Mark II. A Audiometria Tonal Liminar para determinar os limiares auditivos por via aérea nas frequências de $0,25 \mathrm{a} 8 \mathrm{kHz}$, e por via óssea nas frequências de $0,5 \mathrm{a} 4 \mathrm{kHz}$, em cabina acústica (atendendo à norma ANSI S3.1-1991 de nível de ruído ambiental). De modo a determinar o Limiar de Reconhecimento de Fala (LRF) e o Índice Percentual de Reconhecimento de Fala (IPRF), foi realizada a Logoaudiometria, por meio de palavras dissílabas devido à baixa compreensão da paciente com palavras monossilábicas. Tais procedimentos foram realizados no audiômetro Interacoustics, modelo AD229. Realizou-se também Imitanciometria, composta por timpanometria e pesquisa dos reflexos acústicos contralaterais; a mesma foi realizada nas frequências de 500, 1000, 2000 e $4000 \mathrm{~Hz}$, para avaliar a integridade da orelha média, bem como a verificação central por meio do arco reflexo, com o equipamento Interacoustics, modelo AT235. Para a classificação dos limiares auditivos, utilizou-se classificação da Organização Mundial da Saúde (1997).

Os resultados dos achados fonoaudiológicos pré-treinamento auditivo corroboravam com a queixa da paciente, evidenciando uma perda auditiva neurossensorial de grau leve, curva timpanométrica do tipo A e ausência dos reflexos acústicos contra laterais em ambas as orelhas. Na logoaudiometria foi observada apenas $12 \%$ de IPRF, bilateralmente, em intensidade adequada e confortável.

Realizou-se também o Potencial Evocado Auditivo de Tronco Encefálico (PEATE), de modo a avaliar a integridade da via auditiva em nível de Tronco Encefálico. Para o registro do PEATE foi utilizado o módulo Smart-EP da marca Intelligent Hearing Systems ${ }^{\circledR}$, de dois canais. A higienização da pele da adolescente foi realizada com gaze e pasta abrasiva da marca $N U P R E P 囚$, a fim de permitir impedância dos eletrodos com a pele. Os quatro eletrodos foram fixados com pasta condutiva eletrolítica, sendo o eletrodo ativo colocado na fronte $(\mathrm{Fz})$, o terra $(\mathrm{Fpz})$ lateralmente na fronte, e os de referência na mastoide esquerda (T3) e mastoide direita (T4), de acordo com o padrão Internacional 10/20. Utilizou-se fones de inserção para a apresentação dos estímulos e analisou-se a impedância dos eletrodos, antes do início do exame(abaixo de 3 ohms). O registro do PEATE foi monoaural, iniciado com o estímulo clique na intensidade de $80 \mathrm{~dB}$ nHL. Em seguida as respostas foram registradas nas intensidades de 90 e $99 \mathrm{~dB}$ nHL. Realizou-se a reprodutibilidade do traçado para analisar a presença/ausência das ondas. Em relação aos parâmetros da aquisição dos traçados, utilizou-se o 2048 estímulos clique, de $100 \mu \mathrm{seg}$ de duração, com polaridade rarefeita; a taxa de apresentação do estímulo foi de 27,7 estímulos por segundo, utilizou-se filtros de $100-3000 \mathrm{~Hz}$ e aceitou-se no máximo $10 \%$ de artefatos. Foi observada a ausência das ondas I, III e V, na intensidade máxima do equipamento, à direita e à esquerda.

Após a análise dos resultados, a paciente foi encaminhada para terapia no Laboratório de Treinamento Auditivo da Instituição em questão. Devido ao desempenho apresentado no teste de reconhecimento de fala, não foi possível realizar a bateria comportamental de avaliação do processamento auditivo. Contudo, sabendo dos benefícios do treinamento auditivo, como a melhora da plasticidade neuronal, aprimoramento das habilidades auditivas e consequentemente dos comportamentos auditivos, foi escolhido este modelo de intervenção terapêutica, visando minimizar as queixas e dificuldades relatadas na anamnese, em um período pré-operatório ${ }^{5,6}$. A conduta médica também foi o encaminhamento para a cirurgia de remoção do neurinoma e tumores, porém por se tratar de um hospital público, o sujeito em questão aguardava vaga para tal procedimento. Desta forma, realizar o TA foi uma forma de disponibilizar uma abordagem terapêutica imediata.

Antes de iniciar a terapia propriamente dita, foi realizado um teste de consciência fonológica, a fim de avaliar a habilidade do sujeito em operar a linguagem como um objeto, por meio do processamento fonológico no nível silábico e fonêmico. Os resultados foram analisados pela somatória dos pontos em cada nível, os quais evidenciaram 53 acertos num total de 70 possibilidades, sendo 34 no nível silábico e 19 no nível fonêmico.

Após esta etapa, escolheu-se seguir o protocolo de treinamento auditivo computadorizado, por meio do software Escuta Ativa ${ }^{7}$, levando em consideração as características individuais da paciente. Este programa é composto por 12 tarefas com o objetivo de aprimorar as habilidades do processamento auditivo, sendo elas a interação binaural, figura-fundo, resolução temporal, padronização temporal, discriminação, integração e separação binaural. Ao iniciar o programa, é realizado um cadastro e calibração, de forma a atender às carac- 
terísticas individuais dos pacientes. As sessões de terapia seguem uma hierarquia de tarefas, trabalhando diferentes habilidades auditivas em diferentes níveis de complexidade a cada sessão, com a possibilidade de inserção de ruído competitivo a fim de dificultar o treinamento. Foram realizadas 16 sessões, duas vezes por semana, com 30 minutos de duração cada. Destas, 12 sessões destinaram-se às tarefas do software, e as outras quatro sessões às habilidades específicas disponibilizadas pelo software - interação binaural, figura-fundo e discriminação auditiva - às quais o paciente apresentou maior dificuldade 7 . Utilizou-se fone de ouvido supra auricular da marca Sony, modelo MDR-ZX100. Foi realizada uma atividade por sessão, conforme a seguinte ordem de apresentação ${ }^{8}$ (Quadro 1)

Quadro 1. Atividades propostas, habilidade auditiva estimulada, processo gnósico envolvido e breve explicação das tarefas

\begin{tabular}{|c|c|c|c|}
\hline Atividade & $\begin{array}{c}\text { Habilidade auditiva } \\
\text { estimulada }\end{array}$ & $\begin{array}{c}\text { Processo } \\
\text { gnósico }\end{array}$ & Funcionamento \\
\hline Siga a flauta & $\begin{array}{l}\text { Ordenação temporal } \\
\text { (Padrão de duração) } \\
\text { Atenção e Memória }\end{array}$ & $\begin{array}{c}\text { Gnosia não } \\
\text { verbal }\end{array}$ & $\begin{array}{l}\text { Foram apresentados sons de longa e curta duração } \\
\text { e solicitou-se que a criança reproduzisse a mesma } \\
\text { sequência escutada. Níveis fácil e médio com } \\
\text { apresentação de três sons, difícil com quatro sons e } \\
\text { insano com cinco sons. }\end{array}$ \\
\hline Siga o piano & $\begin{array}{l}\text { Padronização Temporal } \\
\text { (Padrão de frequência) } \\
\text { Atenção e Memória }\end{array}$ & $\begin{array}{c}\text { Gnosia não } \\
\text { verbal }\end{array}$ & $\begin{array}{l}\text { Foram apresentados sons de intensidades diferentes } \\
\text { (agudo ou grave) e solicitou-se que a criança } \\
\text { reproduzisse a mesma sequência escutada. Níveis fácil } \\
\text { e médio com apresentação de três sons, difícil com } \\
\text { quatro sons e insano com cinco sons. }\end{array}$ \\
\hline $\begin{array}{l}\text { Quantos } \\
\text { intervalos }\end{array}$ & $\begin{array}{l}\text { Resolução temporal } \\
\text { Atenção }\end{array}$ & Decodificação & $\begin{array}{l}\text { Foram apresentados sons e intervalos e, nesta } \\
\text { atividade, a criança, sempre que percebesse o } \\
\text { intervalo, devia clicar em um número ou, ao final da } \\
\text { sequência, apenas no número correspondente ao total } \\
\text { de intervalos escutados. }\end{array}$ \\
\hline Quantos sons & $\begin{array}{l}\text { Ordenação temporal } \\
\text { Atenção }\end{array}$ & $\begin{array}{l}\text { Gnosia não } \\
\text { verbal }\end{array}$ & $\begin{array}{l}\text { Foram apresentados sons de instrumentos musicais } \\
\text { em diferentes sequências e solicitou-se que a criança } \\
\text { dissesse quantas vezes ouviu o som. }\end{array}$ \\
\hline Qual som ouviu & $\begin{array}{l}\text { Detecção e } \\
\text { discriminação } \\
\text { Atenção } \\
\end{array}$ & Ordenação & $\begin{array}{l}\text { Foram apresentados dois sons verbais e uma pergunta } \\
\text { referente ao que se ouviu (as palavras foram iguais } \\
\text { ou diferentes). }\end{array}$ \\
\hline $\begin{array}{l}\text { Siga a } \\
\text { sequência }\end{array}$ & $\begin{array}{l}\text { Associação } \\
\text { Memória auditiva para } \\
\text { sons não verbais } \\
\text { Atenção }\end{array}$ & Organização & $\begin{array}{l}\text { Foram apresentados sons de animais. A criança devia } \\
\text { memorizá-los e organizá-los, conforme a ordem } \\
\text { solicitada. Níveis fácil e médio: coloque em ordem } \\
\text { alfabética os nomes dos animais ouvidos, coloque } \\
\text { em ordem alfabética inversa os nomes dos animais } \\
\text { ouvidos, diga apenas o terceiro som ouvido etc. Para } \\
\text { tal atividade, as crianças tiveram auxilio visual de uma } \\
\text { trilha do alfabeto. Níveis difícil e insano: ouvia-se uma } \\
\text { história e, ao final, era apresentada uma pergunta que } \\
\text { a criança devia interpretar. }\end{array}$ \\
\hline $\begin{array}{l}\text { Audição e } \\
\text { atenção }\end{array}$ & $\begin{array}{l}\text { Reconhecimento } \\
\text { Fechamento auditivo } \\
\text { Atenção }\end{array}$ & Decodificação & $\begin{array}{l}\text { Foram apresentadas duas palavras auditivamente e } \\
\text { por escrito e a criança devia responder conforme o } \\
\text { solicitado no enunciado (as palavras rimam? começam } \\
\text { com o mesmo som? possuem o mesmo número de } \\
\text { sílabas? etc). Níveis difícil e insano: as palavras escritas } \\
\text { e as escutadas eram diferentes. }\end{array}$ \\
\hline Bem na mira & $\begin{array}{l}\text { Separação binaural } \\
\text { Atenção }\end{array}$ & Codificação & $\begin{array}{l}\text { Foram apresentadas duas palavras ao mesmo tempo, } \\
\text { uma em cada orelha, e solicitou-se que a criança } \\
\text { identificasse a palavra alvo e apontasse de qual lado } \\
\text { foi apresentada. A palavra alvo podia ser dita antes } \\
\text { ou depois da apresentação. }\end{array}$ \\
\hline $\begin{array}{l}\text { Esquerda } \\
\text { Direita }\end{array}$ & Integração binaural & Codificação & $\begin{array}{l}\text { Foram apresentadas palavras, ora de um lado, ora } \\
\text { de outro, e a criança devia identificar quais foram as } \\
\text { palavras e de que lado saiu cada uma. Nível fácil: uma } \\
\text { palavra de cada lado, nível médio: duas, nível difícil: } \\
\text { três e insano: quatro. }\end{array}$ \\
\hline
\end{tabular}




\begin{tabular}{|c|c|c|l|}
\hline Atividade & $\begin{array}{c}\text { Habilidade auditiva } \\
\text { estimulada }\end{array}$ & $\begin{array}{c}\text { Processo } \\
\text { gnósico }\end{array}$ & \multicolumn{1}{c|}{ Funcionamento } \\
\hline Binaural & $\begin{array}{c}\text { Localização } \\
\text { Atenção } \\
\text { Memória }\end{array}$ & Decodificação & $\begin{array}{l}\text { Foram apresentados sons de instrumentos musicais, } \\
\text { ora de um lado, ora de outro, ora longe, ora perto, } \\
\text { e a criança devia identificar de que localização saiu } \\
\text { cada som. Níveis fácil e médio: a resposta podia } \\
\text { ser dada enquanto os estímulos iam sendo ouvidos. } \\
\text { Níveis médio e insano: a resposta foi dada após a } \\
\text { apresentação da sequência, solicitando memorização. }\end{array}$ \\
\hline $\begin{array}{c}\text { Pegue se puder } \\
\text { (Faixa bônus) }\end{array}$ & Atenção & $\begin{array}{l}\text { Foi apresentada uma bandeja de frutas e a criança devia } \\
\text { clicar apenas na maçã que ficava se movimentando. } \\
\text { Níveis fácil e médio: apenas maçã na bandeja mudando } \\
\text { de velocidade ou mudando de tamanho. Nível difícil: } \\
\text { foram adicionadas outras frutas e a criança devia } \\
\text { buscar apenas a maçã. Nível insano: diferentes } \\
\text { frutas na bandeja e ouviam-se pedidos de fregueses; } \\
\text { conforme solicitação do enunciado, a criança devia } \\
\text { clicar na fruta que estava piscando ou na fruta } \\
\text { solicitada auditivamente. }\end{array}$ \\
\hline $\begin{array}{c}\text { Siga o ritmo } \\
\text { (Faixa bônus) }\end{array}$ & Codificação \\
\hline
\end{tabular}

Fonte Melo et al, 2016.

Após o período de intervenção, todos os exames aplicados na avaliação foram reaplicados com o intuito de analisar o efeito do treinamento auditivo. Observou-se na logoaudiometria um IPRF de $32 \%$ à direita e $28 \%$ à esquerda. No PEATE observou-se manutenção da ausência das ondas I, III e V. Enquanto que na avaliação da consciência fonológica foi obtida uma pontuação de 63 pontos no total de 70 , sendo 38 para o nível silábico e 29 para o nível fonêmico.

\section{Discussão}

Sabe-se da importância dos achados médicos e do prognóstico reservado no que se refere à preservação da audição, após remoção do neurinoma em muitos casos de Neurofibromatose tipo 2. Sobretudo, o foco do presente relato de caso foi descrever os efeitos do TA computadorizado em uma adolescente diagnosticada com tal patologia; assim, o TA foi uma abordagem terapêutica paliativa, antes da intervenção cirúrgica, e que buscou minimizar de forma mais rápida as queixas da paciente em relação à compreensão de fala.

Os achados do estudo em questão corroboram com a literatura ao afirmar que o treinamento auditivo é eficaz na melhora da percepção auditiva, pois envolve um conjunto de tarefas referentes à linguagem, cognição e habilidades auditivas ${ }^{9,10}$. Estas são direcionadas para a ativação do sistema auditivo e sistemas associados, de forma a aprimo- rar as funções auditivas originadas pelo sistema nervoso central, em especial na etiologia de base deste estudo, a qual compromete as estruturas elementares para o processamento do som a nível central como o nervo auditivo ${ }^{11}$.

Localizou-se apenas um estudo na literatura compulsada que envolveu TA e $\mathrm{NF}^{11}$, no qual foram realizadas oito sessões de Treinamento Auditivo Acusticamente Controlado, uma vez por semana, com duração de 50 minutos cada, em pacientes com NF1 e TPA. Os autores concluíram que tal intervenção foi eficaz para a melhora das habilidades auditivas de fechamento auditivo, figura-fundo e resolução temporal; e os benefícios obtidos depois do TA se mantiveram após um ano do seu término na população estudada. Apesar de ter sido realizado em outro tipo de NF é possível apontar que tais dados corroboram com os do presente estudo, pois após a intervenção proposta observou-se evolução em todos os achados; no IPRF a paciente apresentou melhora no reconhecimento de fala após o treinamento auditivo demonstrando uma melhora na percepção/discriminação dos sons da fala. Estudos mostram que tal intervenção é capaz de melhorar as habilidades de figura-fundo e fechamento auditivo ${ }^{11,12}$. Sabe-se que a codificação necessária ao teste do IPRF é a mesma para as habilidades citadas; dessa forma, utilizam-se os mesmos mecanismos de processamento destes estímulos, e deste modo pode-se afirmar que os achados são em detrimento destes dados. 
Do mesmo modo, em relação à consciência fonológica, a paciente apresentou um desempenho superior em relação ao pré-treinamento, sendo mais evidente no nível fonêmico. Isso sugere que as habilidades em manipular e operar os sons da fala foram aperfeiçoadas, demonstrando que a paciente estava mais atenta auditivamente, especialmente às solicitações do pesquisador. Estes achados corroboram com a literatura, ao afirmar que a consciência fonológica é sensível ao TA, uma vez que a discriminação de sílabas e fonemas é constantemente estimulada, bem como a estrutura dos sons da língua, favorecendo o processamento fonológico?.

A ausência de ondas no PEATE sugere que os estímulos apresentados não foram eficazes para conduzir e desencadear respostas das estruturas centrais avaliadas por este potencial, com estímulo clique. Este fato sugere que as mudanças eletrofisiológicas não sofreram influência a partir do número de sessões propostas, ou seja, o período de intervenção não foi suficiente para promover uma plasticidade neuronal significativa no registro deste potencial. Os dados concordam com os achados de outro estudo, que analisou o Potencial Evocado Auditivo de Longa Latência (PEALL) em idosos após tal intervenção ${ }^{10}$. Por outro lado, diverge de outro estudo que revela resultados melhores, referentes à latência e amplitude das ondas avaliadas, tanto para o PEATE, quanto para o PEALL, sendo que este último não foi avaliado no presente estu$\mathrm{do}^{13}$. Na época do atendimento desta paciente em questão, não se tinha disponível o PEALL, desta forma, aponta-se aqui uma possível limitação do presente estudo.

Outra hipótese que justifica a ausência de ondas no PEATE pode decorrer unicamente da etiologia de base, uma vez que esta, ao promover lesão nas estruturas centrais, como o VIII par craniano - o qual é avaliado pela onda I deste potencial - não permite o registro da resposta e a condução do estímulo para as estruturas mais altas do tronco encefálico, como o complexo olivar superior e o lemnisco lateral, avaliados respectivamente pelas ondas III e $\mathrm{V}^{13}$. Supõe-se então, que com a intervenção cirúrgica para a retirada do tumor, poderia haver a presença das ondas, por mais que estas não estivessem morfologicamente adequadas.

Apesar da subjetividade, o relato da paciente endossa o que os achados deste estudo revelaram; a intervenção foi significativa para suas atividades de vida diária, uma vez que houve melhora na compreensão de fala, permitindo maior qualidade de vida pré-intervenção cirúrgica. Além disso, a paciente referiu interesse em continuar as sessões depois da retirada dos tumores, pois percebeu que a TA trouxe benefícios para sua comunicação e, consequentemente, para sua qualidade de vida. $\mathrm{O}$ tema qualidade de vida em pacientes com NF2 ${ }^{14}$ já foi apontado como algo preocupante, pois os sintomas clínicos significativos desta patologia trazem reflexos importantes à vida destes pacientes.

Cabe ressaltar que tal intervenção foi complementar ao tratamento médico e mostrou-se, neste caso específico, uma alternativa de reabilitação da comunicação, enquanto a adolescente estudada aguardava o processo cirúrgico já anteriormente indicado pelo médico responsável.

Sabe-se que outras abordagens para a intervenção nos casos de Neurofibromatose tipo 2 são citadas na literatura, como por exemplo, o Implante Auditivo $^{15}$ de Tronco Cerebral após a cirurgia de remoção dos tumores, quando há um grande prejuízo da audição; e o uso de novas terapias medicamentosas eficazes para pacientes ${ }^{16,17}$ com NF2, que funcionam como inibidores de crescimento endotelial vascular reduzindo o crescimento do schwannoma e dos tumores em alguns pacientes. Entretanto, neste estudo, hipotetizou-se que o treinamento auditivo traria um efeito positivo na comunicação da paciente e tal concretizou-se, evidenciando a importância de novos estudos com arranjos amostrais mais robustos para comprovar essa hipótese.

\section{Conclusão}

Constataram-se efeitos positivos do treinamento auditivo neste caso de NF2, pois permitiu uma melhora na percepção auditiva capaz de influenciar na qualidade de vida do sujeito. Sugere-se ainda que estudos sejam realizados nesta mesma perspectiva, já que não foram encontradas pesquisas específicas com treinamento auditivo nessa patologia nas bases de dados compulsadas.

\section{Referências bibliográficas}

1. Yin, L. et al. Unilateral Vestibular Schwannomas in Childhood without Evidence of Neurofibromatosis: Experience of 10 Patients at a Single Institute. Pediatric Unilateral Acoustic Neuromas. 2016; 13(4):318-22. 
2. Silva M., Romão J., Paiva A.D. Neurofibromatosis type ii: case reports. Revista Portuguesa de ORL, 2016; 54 (4) 187-191.

3. Faria, E.F. et al. Schwannoma de Acústico: Revisão Bibliográfica. Rev Pat Tocantins, 2015; 2(2): 16-22.

4. Batista P. B, Lemos S. M. A., Rodrigues L.O.C, Rezende N.A. Auditory temporal processing deficits and language disorders in patients with neurofibromatosis type 1. J Commun Disord. 2014; 48:18-26.

5. Musiek F., Shinn J., Hare C. Plasticity, auditory training, and auditory processing disorders. Semin Hear. 2002; 23(4): 263-76.

6. Weihing J., Chermak G.D, Musiek F.E. Auditory training for central auditory processing disorder. Semin Hear. 2015; 36(04): 199-215.

7. Alvarez, A., Sanchez, M. L., Guedes, M. C. Escuta Ativa Avaliação e Treinamento Auditivo Neurocognitivo [software]. Pato Branco, PR: CTS Informática; 2010.

8. Melo, Â., et al . Efeitos do treinamento auditivo computadorizado em crianças com distúrbio do processamento auditivo e sistema fonológico típico e atípico. Audiol., Commun. Res. 2016; 21: 1683.

9. Calarga, K.S. Tradução de adaptação de um software de treinamento auditivo para escolares [Tese de mestrado em Ciências da Reabilitação]. São Paulo (SP): Universidade Federal de São Paulo; 2016.
10. Morais A. A, Rocha-Muniz C. N., Schochat E. Efficacy of Auditory Training in Elderly Subjects. Front Aging Neurosci. 2015; 7: 78 .

11. Batista B. P. Treinamento auditivo acusticamente controlado em pacientes com neurofibromatose tipo 1. [tese] Minas Gerais(MG): Universidade Federal de Minas Gerais; 2016.

12. Ávila R. R. A, Murphy C. F. B, Schochat E. Efeitos do Treinamento Auditivo em Idosos com Comprometimento Cognitivo Leve. Psicol. Reflex. e Crít. 2014; 27(3): 547-55.

13. Figueiredo C.C. et al. Behavioral and electrophysiological auditory processing measures in traumatic brain injury after acoustically controlled auditory training: a long-term study. Einstein. 2015; 13(4): 535-40.

14. Rosalie E. et al. Longitudinal evaluation of quality of life in 288 patients with neurofibromatosis 2. J Neurol. 2014; 261(5): 963-969.

15. Fernandes, N. F. et al . Satisfação e qualidade de vida em usuários de implante auditivo de tronco cerebral. CoDAS, 29(2), e 20160059.

16. Jaishri O. et al. Consensus Recommendations for Current Treatments and Accelerating Clinical Trials for Patients with Neurofibromatosis Type 2. Am J Med Genet A. 2012 Jan; 158A(1): 24-41.

17. Jaishri O. et al. Efficacy and Biomarker Study of Bevacizumab for Hearing Loss Resulting From Neurofibromatosis Type 2Associated Vestibular Schwannomas J Clin Oncol. 2016 May 10; 34(14): 1669-1675. 